\title{
Prognostic implication of morphology, cyclinE2 and proliferation in EBV-associated T/NK lymphoproliferative disease in non-immunocompromised hosts
}

Siok-Bian Ng ${ }^{1,8^{*}}$, Koichi Ohshima ${ }^{2}$, Viknesvaran Selvarajan ${ }^{3}$, Gaofeng Huang ${ }^{4}$, Shoa-Nian Choo ${ }^{3}$, Hiroaki Miyoshi ${ }^{2}$, Shi Wang ${ }^{3}$, Hsin-Chieh Chua ${ }^{5}$, Allen Eng-Juh Yeoh ${ }^{5}$, Thuan-Chong Quah ${ }^{5}$, Liang-Piu Koh ${ }^{6}$, Poh-Lin Tan ${ }^{5}$ and Wee-Joo $\mathrm{Chng}^{7^{*}}$

\begin{abstract}
Background: EBV-associated T/NK-cell lymphoproliferative diseases (TNKLPD) is a rare spectrum of disease that occurs more commonly in Asia, and Central and South America. It commonly affects children and young adults and is an aggressive disease that is poorly understood with no known biologic markers that can predict prognosis. The systemic form of TNKLPD includes chronic active EBV infection of T/NK type, aggressive NK cell leukemia and systemic EBV + T-cell lymphoproliferative disease of childhood.

Methods: In this study, we analyse the clinicopathologic and genetic features of 22 cases of systemic TNKLPD in non-immunocompromised patients, including chronic active EBV infection of T/NK cell type and systemic EBV + T-cell lymphoproliferative disease of childhood. We also performed gene expression profiling in a subset of cases to identify markers that may be of prognostic relevance and validated our results using immunohistochemistry.

Results: The median age is 14.9 years and two of our 22 cases occurring in patients older than 30 years. Fifteen of 17 cases (88\%) with adequate data were of T-cell origin. Eleven of 22 cases revealed polymorphic cellular infiltrate (P-group) while the rest showed monomorphic lymphoid infiltrate (M-group). We found a significant difference in survival between P-group vs M-group patients with median survival not yet reached in P-group, and 1 month in M-group ( $p=0.0001$ ), suggesting a role for morphology in predicting patient outcome. We also performed gene expression profiling in a subset of patients and compared the genes differentially expressed between P-group and M-group cases to identify markers of prognostic value. We identified cyclin E2 gene and protein to be differentially expressed between patients with good outcome (P-group, median expression 8\%) and poor outcome (M-group, median expression 42\%) ( $p=0.0005)$. In addition, the upregulation of cyclin E2 protein in M-group cases correlated with a higher Ki67 proliferation rate (Pearson correlation $r=0.73, p=0.0006$ ) detected by immunohistochemistry. High cyclin E2 expression was also significantly associated with shorter survival $(p=0.0002)$.

(Continued on next page)
\end{abstract}

\footnotetext{
* Correspondence: patnsb@nus.edu.sg; mdccwj@nus.edu.sg

'Department of Pathology, National University Cancer Institute of Singapore, National University Health System, Yong Loo Lin School of Medicine, Cancer Science Institute of Singapore, National University of Singapore, Singapore, Singapore

${ }^{7}$ Department of Haematology-Oncology, National University Cancer Institute of Singapore, National University Health System, Yong Loo Lin School of Medicine, Cancer Science Institute of Singapore, 1E, Kent Ridge Rd, Singapore 119228, Singapore

Full list of author information is available at the end of the article
}

\section{Biomed Central}

(c) 2014 Ng et al.; licensee BioMed Central Ltd. This is an Open Access article distributed under the terms of the Creative Commons Attribution License (http://creativecommons.org/licenses/by/4.0), which permits unrestricted use, distribution, and reproduction in any medium, provided the original work is properly credited. The Creative Commons Public Domain Dedication waiver (http://creativecommons.org/publicdomain/zero/1.0/) applies to the data made available in this article, unless otherwise stated. 
(Continued from previous page)

Conclusion: Our data suggests the potential role of monomorphic morphology, high cyclin E2 and Ki67 expression as adverse prognostic factors for TNKLPD.

Keywords: EBV-associated T/NK lymphoproliferative disease, Molecular signature, Morphology, Cyclin E2, Proliferation, Prognosis

\section{Background}

The term EBV-associated T/NK-cell lymphoproliferative disorders (TNKLPD) encompasses a spectrum of disease entities in non-immunocompromised patients characterised by an Epstein-Barr virus (EBV)-infected, cytotoxic $\mathrm{T}$ or NK-cell proliferation that is polyclonal or monoclonal. The systemic form of TNKLPD includes chronic active EBV infection of T/NK-cell type (CAEBV), aggressive NK cell leukemia (ANKL) and systemic EBV-positive Tcell lymphoproliferative disease of childhood (STLPDC) [1-4]. TNKLPD is rare in the Western population and occurs with increased frequency in Asians, Native Americans from Central and South America and Mexico $[1,2]$ Morphologically, it is characterized by a wideranging cytological appearance from reactive appearance to overt leukemia/lymphoma [5-7]. The diagnosis of TNKLPD can be challenging because cytologic features may be deceptively benign despite the frequently aggressive clinical behaviour.

Historically, the terminology and classification of TNKLPD have been problematic as this process has been described under a variety of terms including: fulminant EBV + T-cell lymphoproliferative disease (LPD) of childhood [8], fatal infectious mononucleosis; fulminant hemophagocytic syndrome in children in Taiwan [9], fatal EBV-associated hemophagocytic syndrome in Japan [10] and severe CAEBV [11]. However, recent efforts have been made to achieve international consensus on the nomenclature and classification of TNKLPD and it is now recognized that most of the EBV-associated T/NK LPD in immunocompetent children and young adults with systemic presentation belong to the same spectrum of disease, including CAEBV and STLPDC. A consensus meeting was held at the National Institute of Health (NIH) in 2008 and it was recommended that the term CAEBV should be applied to systemic LPDs that are not frank lymphomas and that arise during primary infection and persist for over 6 months. On the other hand, the term 'systemic EBV-positive T-cell LPD', as adopted by the WHO classification, is the preferred pathologic designation over CAEBV for those cases that are clonal with an aggressive clinical course and require aggressive treatment [12]. A clinicopathological categorization of TNKLPD was proposed by Ohshima et al. in 2008 [13]. According to the proposal, EBV-associated T/NK LPD in children can be categorised into 4 groups (A1, A2, A3 and B) based on morphology of the infiltrate, clonality and clinical presentation. A consensus report following the 4th Asian Hematopathology Workshop on the classification and terminology of TNKLPD was also recently published [14]. In this report, TNKLPD can be divided into systemic and cutaneous forms (see Additional file 1, for a summary of the proposed classifications of TNKLPD). The systemic form includes CAEBV-T/NK, ANKL and STLPDC.

In this study, we analysed the clinicopathologic and genetic features of 22 cases of systemic form of TNKLPD in Singapore and Japan using the criteria recommended by WHO and NIH in 2008, including CAEBV and STLPDC. We found that patients with polymorphic infiltrate (Pgroup) have a significantly better outcome than cases with monomorphic infiltrate (M-group). We then performed, for the first time, gene expression profiling (GEP) in a subset of patients and compared the gene signature between P-group and M-group patients with good and poor outcome, respectively, in order to identifying targets that may be important in predicting outcome.

\section{Methods}

\section{Case selection and definition}

Patients with a diagnosis of EBV-associated T/NK-cell lymphoproliferative disorder without known underlying immune deficiency were identified from the archives of the Department of Pathology, National University Hospital (NUH), and Kurume University, from 2003 to 2013. Cases were reviewed, additional immunohistochemistry and in-situ hybridization for EBV-encoded small RNA (EBER) were performed and the cases were classified according to the 2008 WHO lymphoma classification and nomenclature proposed following the NIH consensus report in 2008 (Table 1) [12]. The determination of $\mathrm{T}$ vs NK cell lineage is based on the TCRG gene clonality and protein expression of TCR $\alpha \beta$ and TCR $\gamma \delta$. Extranodal nasaltype NK/T-cell lymphoma cases were excluded. A total of 22 cases with adequate material for workup and fulfilled the diagnostic criteria were identified and included CAEBV of T/NK type, and systemic EBV-associated Tcell LPD of childhood. The clinical history, previous biopsies and laboratory results were reviewed for evidence of hemophagocytic lymphohistiocytosis (HLH). Hemophagocytic lymphohistiocytosis (HLH) is defined by at least five 
Table 1 Pathologic and genetic features and classification of EBV + T/NK-cell lymphoproliferative disease

\begin{tabular}{|c|c|c|c|c|c|c|c|c|c|c|c|}
\hline No & Diagnosis* & Ohshima category** & Histo & CD4 & CD8 & CD56 & TCR $\alpha \beta$ & TCR $\gamma \delta$ & TCR PCR & Cell of origin & BM karyotype \\
\hline 1 & CAEBV & $\mathrm{A} 1$ & $P$ & - & - & - & - & - & PC & TCR silent vs NK & $\mathrm{N}$ \\
\hline 2 & CAEBV & $\mathrm{A} 1$ & $P$ & - & + & - & + & - & PC & $\mathrm{T}$ & N \\
\hline 3 & CAEBV & $\mathrm{A} 1$ & $P$ & - & + & + & - & - & $P C$ & TCR silent vs NK & $\mathrm{N}$ \\
\hline 4 & STLPDC & $\mathrm{A} 2$ & $P$ & - & + & - & + & - & $M C$ & $T$ & $N$ \\
\hline 5 & STLPDC & $\mathrm{A} 2$ & $P$ & - & + & - & - & - & $M C$ & T & N \\
\hline 6 & STLPDC & $\mathrm{A} 2$ & $P$ & - & + & - & - & - & $M C$ & T & $\mathrm{N}$ \\
\hline 7 & STLPDC & $\mathrm{A} 2$ & $P$ & + & - & + & Focal + & - & $M C$ & T & $\mathrm{N}$ \\
\hline 8 & CAEBV vs STLPDC & $\mathrm{A} 1$ or $\mathrm{A} 2$ & $P$ & NA & NA & + & NA & NA & NA & NA & NA \\
\hline 9 & CAEBV vs STLPDC & $\mathrm{A} 1$ or $\mathrm{A} 2$ & $P$ & - & + & - & NA & NA & NA & NA & NA \\
\hline 10 & CAEBV vs STLPDC & $\mathrm{A} 1$ or $\mathrm{A} 2$ & P & + & + & - & NA & NA & NA & NA & NA \\
\hline 11 & CAEBV vs STLPDC & $\mathrm{A} 1$ or $\mathrm{A} 2$ & $P$ & NA & NA & - & NA & NA & NA & NA & NA \\
\hline 12 & STLPDC & $\mathrm{A} 3$ & M & - & + & + & Focal + & - & $M C$ & T & Abn \\
\hline 13 & STLPDC & A3 & M & - & + & - & + & - & $M C$ & T & N \\
\hline 14 & STLPDC & B & M & - & + & + & + & - & $M C$ & T & Abn \\
\hline 15 & STLPDC & B & M & - & + & - & - & - & $M C$ & T & Abn \\
\hline 16 & STLPDC & B & M & + & - & - & Focal + & - & PC & favour $T$ & Abn \\
\hline 17 & STLPDC & B & M & - & + & - & + & - & $M C$ & T & N \\
\hline 18 & STLPDC & B & M & - & + & - & + & - & $M C$ & T & Mosaic \\
\hline 19 & STLPDC & B & M & - & + & - & + & - & NA & T & N \\
\hline 20 & STLPDC & B & M & + & - & - & + & NA & $P C$ & T & Abn \\
\hline 21 & STLPDC & B & M & - & - & - & NA & NA & $N A^{\ddagger}$ & NA & NA \\
\hline 22 & favour STLPDC & $A 3$ or $B$ & M & - & - & + & + & - & NA & $\mathrm{T}$ & NA \\
\hline
\end{tabular}

Abbreviations: Histo histology, $M$ monomorphic, $P$ polymorphic, $T C R$ clonality for T-Cell Receptor Gamma gene by PCR, MC monoclonal, $P C$ polyclonal, Abn abnormal, $N$ normal, $N A$ not available, OS overall survival, $B M$ bone marrow, CAEBV chronic active EBV infection of T-NK type, STLPDC systemic EBV + T cell lymphoproliferative disorder of childhood, EBV + TLPD EBV-positive T cell lymphoproliferative disorder.

${ }^{\ddagger} E B V$ terminal repeats analysis revealed monoclonality.

*Based on WHO classification and recommendation from NIH consensus meeting in 2008 [12]

**Based on categorization of EBV + T/NK lymphoproliferative disorders in children by Ohshima et al. [13].

of the eight criteria including fever, splenomegaly, bicytopenia, hypertriglyceridemia, and/or hypofibrinogenemia, hemophagocytosis, low/absent NK-cell-activity, hyperferritinemia, and high-soluble interleukin-2-receptor levels [15].

Based on the WHO classification and NIH recommendation [1,12], the term 'CAEBV' is applied to systemic LPDs that are not frank lymphomas and that arise during primary infection and persist for over 6 months. The term 'systemic EBV-positive T-cell LPD', as adopted by the WHO classification, is used for those cases that are monoclonal. We also categorized the cases based on the criteria proposed by Ohshima et al. [13] (ie. morphology, clonality and clinical presentation) into the following (i) category A1, polymorphic and polyclonal LPD; (ii) category A2, polymorphic and monoclonal LPD; (iii) category A3, monomorphic and monoclonal LPD; and (iv) category $\mathrm{B}$, monomorphic and monoclonal LPD with fulminant course (Table 1). We considered the presence of an abnormal karyotype and/or monoclonal TCRG gene rearrangement as indicative of a clonal proliferation [14].
Fulminant course is defined as rapid clinical progression within 1 or 2 months [2]. Follow up data was collected.

Four of the 22 cases of TNKLPD with adequate formalin fixed paraffin embedded (FFPE) tissue and good quality RNA were selected for GEP (cases 5, 7, 16, 17, see Table 2). The FFPE tissues used for GEP included lymph node and skin biopsies. FFPE control tissues from normal skin and lymph node were also included. This study is approved by the Domain Specific Review Board of the National Healthcare Group, Singapore.

\section{RNA extraction from FFPE for gene expression profiling}

Total RNA from human FFPE tissues were isolated using High Pure RNA Paraffin Kit (Roche Applied Science, Mannheim Germany) according to the manufacturer's recommendations. To achieve higher recovery of RNA, $10 \mu \mathrm{m}$ FFPE sections were deparaffinized with $100 \%$ xylene followed by $100 \%$ ethanol. The dried tissues were then subjected to proteinase $\mathrm{K}$ digestion and remaining steps were performed as per the manufacturer's protocol. Concentration and purity of total RNA samples were 
Table 2 Clinical, survival and treatment data of EBV + TNK-cell lymphoproliferative disease

\begin{tabular}{|c|c|c|c|c|c|c|c|c|c|c|c|}
\hline No & Ohshima category* & Histo & Sex & Ethnicity & Age (yrs) & Tissue type & GEP & $\mathrm{HLH}$ & Treatment & Follow up & OS (mth) \\
\hline 1 & $\mathrm{~A} 1$ & $P$ & M & Indonesian & 3.4 & $\mathrm{BM}$ & No & + & HLH -P & Alive & 55 \\
\hline 2 & $\mathrm{~A} 1$ & $P$ & M & Chinese & 13.1 & BM & No & + & HLH-P, AHCT & Dead (AHCT complications) & 48 \\
\hline 3 & $\mathrm{~A} 1$ & $P$ & M & Vietnamese & 24.8 & BM & No & + & Chemo & Alive & 6 \\
\hline 4 & A2 & $P$ & $\mathrm{~F}$ & Chinese & 5.3 & BM & No & + & HLH-P, AHCT & Alive & 64 \\
\hline 5 & $\mathrm{~A} 2$ & $P$ & M & Chinese & 1.4 & Skin & Yes & + & HLH-P , AHCT & Alive (graft rejection) & 95 \\
\hline 6 & A2 & $\mathrm{P}$ & $\mathrm{F}$ & Indonesian & 6.1 & BM & No & + & HLH-P & Alive & 31 \\
\hline 7 & A2 & P & M & Chinese & 16.7 & $L N$ & Yes & - & Steroids, AHCT & Dead & 14 \\
\hline 8 & $\mathrm{~A} 1$ or $\mathrm{A} 2$ & $P$ & M & Japanese & 4 & liver & No & + & AHCT & Alive & 94.4 \\
\hline 9 & $\mathrm{~A} 1$ or $\mathrm{A} 2$ & $P$ & $\mathrm{~F}$ & Japanese & 19 & BM & No & - & AHCT & Alive & 9.2 \\
\hline 10 & $\mathrm{~A} 1$ or $\mathrm{A} 2$ & $P$ & $\mathrm{~F}$ & Japanese & 19 & BM & No & + & Chemo & Alive & 32.5 \\
\hline 11 & $\mathrm{~A} 1$ or $\mathrm{A} 2$ & $\mathrm{P}$ & $\mathrm{F}$ & Japanese & 39 & Skin & No & - & Chemo & Dead & 21.8 \\
\hline 12 & $\mathrm{~A} 3$ & M & $\mathrm{F}$ & Vietnamese & 16.4 & BM & No & + & HLH-P & Dead & 0.4 \\
\hline 13 & $\mathrm{~A} 3$ & M & $\mathrm{F}$ & Chinese & 4.8 & $\mathrm{BM}$ & No & + & HLH-P & Alive & 21 \\
\hline 14 & B & M & M & Indonesian & 13.3 & BM & No & + & HLH -P & Dead & 0.83 \\
\hline 15 & B & M & M & Indonesian & 20.9 & BM & No & + & HLH -P & Dead & 2 \\
\hline 16 & B & M & $\mathrm{F}$ & Chinese & 9.5 & LN & Yes & + & Supportive & Dead & 1 \\
\hline 17 & B & M & M & Chinese & 18.7 & LN & Yes & + & HLH -P & Dead & 1.16 \\
\hline 18 & B & M & M & Vietnamese & 1 & BM & No & + & $\mathrm{HLH}-\mathrm{P}$ & NA & NA \\
\hline 19 & B & M & M & Filipino & 7.6 & BM & No & + & $\mathrm{HLH}-\mathrm{P}$ & Dead & 1 \\
\hline 20 & B & M & $\mathrm{F}$ & Chinese & 19.3 & BM & No & + & HLH-P & Dead & 0.1 \\
\hline 21 & B & M & $\mathrm{F}$ & Japanese & 48 & BM & No & + & Chemo, AHCT & Dead & 2.2 \\
\hline 22 & $A 3$ or $B$ & M & M & Others & 18.3 & BM & No & NA & Supportive & Dead & 0.7 \\
\hline
\end{tabular}

Abbreviations: $M$ male, $F$ female, Histo histology, $P$ polymorphic, $M$ monomorphic, HLH-P 2004 Histiocytic Lymphohistiocytosis protocol, AHCT Allogeneic hematopoietic cell transplant, + present, - absent, TCR Clonality for TCRG gene, $R$ rearranged, $N R$ not rearranged, $N A$ not available, OS overall survival, $L N$ lymph node, $B M$ bone marrow, Chemo chemotherapy. *Categorization of EBV-associated T/NK LPD in children proposed by Ohshima et al. [13].

measured using the NanoDrop ND 3.0 spectrophotometer (NanoDrop Technologies Inc, Wilmington, DE). RNA integrity was assessed with the Agilent 2100 Bioanalyzer (Agilent Technologies, Palo Alto, CA) and the RNA 6000 LabChip kit (Agilent Technologies).

\section{Gene expression profiling and analysis}

We conducted a genome-wide gene expression profiling (GEP) on TNKLPD and normal control FFPE samples using the Illumina WG-DASL (Whole Genome cDNAmediated Annealing, Selection, and Ligation) (Illumina, Inc., San Diego) [16,17]. Briefly, $250 \mathrm{ng}$ of total RNA from each sample were converted to biotinylated cDNA using the Illumina TotalPrep RNA Amplification Kit (Ambion, Inc., Austin, TX, USA) according to the manufacturer's recommendations. The cDNA targets were hybridized to HumanRef- 8 Expression BeadChip arrays (Illumina, Inc., San Diego), which contain 24526 probes, for 16 hours before being washed and stained with streptavidin-Cy3 according to the manufacturer's protocol. The beadchips were scanned and quantitated using Illumina's Bead-Station 500GX Genetic Analysis Systems scanner. The raw signals extracted were normalized using a linear calibration method, as described in our previous paper [18]. Analysis of the data was done by $\mathrm{R} /$ Bioconductor.

Since we found a significantly poorer survival in patients with polymorphic infiltrate (P-group) vs patients with monomorphic infiltrate (M-group), we compared genes that are differentially expressed between P-group and M-group to identify genes that are of prognostic value. Significance analysis of microarrays (SAM) [19] was not appropriate due to the small sample size and we selected the genes with more than 4-fold change for comparison between the two groups with good and poor outcome.

\section{Immunohistochemistry (IHC)}

In order to validate the expression of cyclin E2 and Ki67 in the CD3-positive tumor cells and not the reactive population, we performed the following double stains CD3/ cyclin E2 and CD3/Ki67 on four- $\mu$ m sections of TNKLPD samples [including bone marrow (BM), skin and lymph node (LN) biopsies] using the conditions listed in Additional file 2. Appropriate positive tissue controls were used. The stains were performed using Leica 
BondMax auto-stainer. The immunohistochemical expression for the antibodies was manually scored as a percentage of the CD3-positive tumor cell population by one of the authors (NSB), without knowledge of the clinicopathologic and GEP data. Since there is no well-established cutoff criteria for high cyclinE2 expression in the literature, we determined the median value of cyclinE2 expression in our 22 cases and defined high cyclinE2 expression as equal or greater than the median expression. The median expression of cyclinE2 was determined as $15 \%$ in our cases and high cyclinE2 expression is defined as $15 \%$ or more expression in the CD3-positive tumor cell population.

\section{Results}

\section{Clinical and laboratory findings}

The age of the patients ranged from 1 to 48 years old with median age at the time of diagnosis of 14.9 years (Table 2). Two of our cases occurred in patients older than
30 years of age (case 11 and 21) similar to previous observations that this disease can occasionally affect adults $[4,13,14]$. There were 12 males and 10 females. All the cases presented with fever. Hepatomegaly and/or splenomegaly were present in 21 of 22 patients. Nine of 18 patients with adequate data had lymphadenopathy and 21 out of 22 had cytopenias. 18 out of 21 cases fulfilled the criteria of HLH. Polymerase chain reaction (PCR) for EBV was detected in the blood of all 17 cases with adequate data.

\section{Pathologic features}

Microscopically, the biopsies from BM, LN and skin showed a broad spectrum of appearance ranging from polymorphic cellular infiltrate (Figure 1A) composed of abundant reactive cells, to overtly malignant monomorphous lymphoid proliferation (Figure 1B). Hemophagocytosis is evident in the majority of cases. Eleven of 22 cases

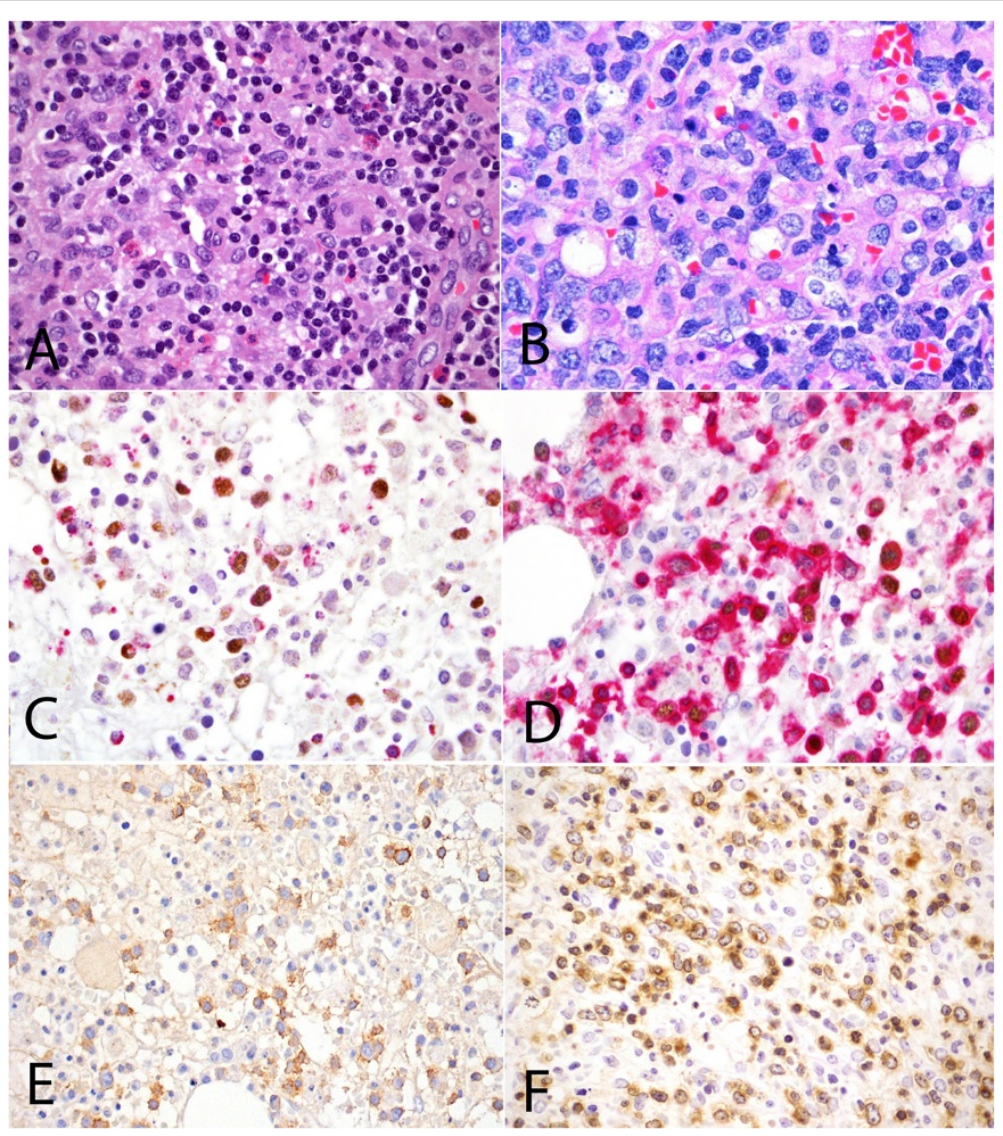

Figure 1 Morphology and phenotype of TNKLPD. Case with polymorphic infiltrate composed of mixed population of small lymphoid cells with minimal atypia, histiocytes and eosinophils (A, H\&E, original magnification 600x). Monomorphic infiltrate consisting of large malignant lymphoid cells with irregular nuclei (B, H\&E, original magnification 600X). Expression of cytotoxic marker TIA1 in EBER-positive tumour cells (C, EBER/TIA1 double stain, EBER stains nucleus brown and TIA1 stains cytoplasmic granules red, original magnification 1000X). Positive expression for EBER in situ hybridization in CD3-positive tumor cells (D, EBER/CD3 double stain, EBER stains nucleus brown and CD3 stains cell membrane/cytoplasm red, original magnification 1000X). Positive expression for CD56 (E, CD56, original magnification 600X) and TCRbeta (F, TCRB, original magnification 600x). All photographs were taken with a DP20 Olympus camera (Olympus, Tokyo, Japan) using an Olympus BX41 microscope (Olympus). Images were acquired using DP Controller 2002 (Olympus) and processed using Adobe Photoshop version 5.5 (Adobe Systems, San Jose, CA, USA). 
revealed polymorphic cellular infiltrate while the rest showed monomorphic lymphoid infiltrate (Table 1).

Phenotypically, all cases revealed a CD3+ cytotoxic T/NK-cell lymphoid infiltrate which expresses EBER by in-situ hybridization (Figures $1 \mathrm{C}$ and D). Of the 20 cases tested, 13 were CD4-/CD8+ and 3 were CD4-/CD8-. Another three were CD4+/CD8- and one case was CD4+ $/ \mathrm{CD} 8+$. Expression of CD56 was present in 6 cases (Figure 1E). TCR $\alpha \beta$ immunoreactivity was seen in 12 of 17 cases (Figure 1F) tested. All cases studied were negative for $\mathrm{TCR} \gamma \delta$ protein. Monoclonal T-cell receptor gamma gene rearrangement was detected in 10 out of 15 patients, of which 4 cases revealed a polymorphic proliferation. Bone marrow cytogenetics was performed in 16 cases, of which 5 revealed abnormal karyotypes.

With regards to cell lineage, 15 of the 17 cases (88\%) with adequate workup were of $\mathrm{T}$ cell lineage; 10 of these 15 cases had clonal rearrangement of TCRG gene by PCR, the remaining 5 cases showed positive expression for TCR $\alpha \beta$. Two cases ( 1 and 3 ) were negative for TCR $\alpha \beta$ and TCR $\gamma \delta$, and TCRG gene was polyclonal. These 2 cases may represent either a TCR-silent or NK phenotype (Table 1).

\section{Categorization of TNKLPD, treatment and clinical outcome}

The diagnosis of the cases based on WHO and NIH proposed nomenclature [12] as well as the corresponding categorization as proposed by Ohshima et al. [13] are provided in Table 1 . Three cases were classified as CAEBV and 15 were STLPDC. The remaining 4 cases were difficult to classify accurately because of lack of clonality data. All the CAEBV cases had polymorphic infiltrate and are polyclonal by definition (Ohshima type A1). Of the 15 STLPDC, 4 cases had polymorphic and monoclonal infiltrate, corresponding to Ohshima type A2. The remaining 11 STLPDC cases showed monomorphic proliferation. Case 12 was categorised as Ohshima type A3 although the patient died 0.4 month after diagnosis because she presented with symptoms for 2 years prior to diagnosis. There were no cases of aggressive NK cell leukemia and all our cases with aggressive clinical course were of $\mathrm{T}$ cell origin.

Follow up was available in 21 cases with median of 11.6 months (ranged from 3 days to 8.9 years). The overall median survival was 21.8 months. Of the 18 cases that could be classified, the median survival for STLPDC was 2 months compared to median survival not yet reached for CAEBV. However, this difference was not significant and may be related to small sample size ( $\mathrm{p}=$ 0.0668).

Interestingly, cases with polymorphic infiltrate (P-group) and those with monomorphic infiltrate (M-group) showed a statistically significant difference in survival with median survival not yet reached in P-group and 1 month in $\mathrm{M}$ group ( $p=0.0001$ ) (Figure 2 ). We did not find any significant difference in survival between cases with polyclonal and monoclonal lymphoid infiltrate, although this may again be due to small sample size.

Five of 11 patients in P-group were treated with 2004 HLH protocol, a chemo-immunotherapy which includes etoposide, dexamethasone, cyclosporine A upfront and, in selected patients, intrathecal therapy with methotrexate and corticosteroids [15] (Table 2). Six P-group cases also received allogeneic hematopoietic cell transplant (AHCT), of which one died of disease and one died from AHCT related complications. The other 4 patients who received AHCT remained alive although one patient had graft rejection. Overall, 8 out of 11 P-group patients remained alive. In contrast, 9 of 10 patients in M-group with follow up data died of disease and only one patient remained alive at 21 months of follow up. Eight of 11 M-group patients were treated with 2004 HLH protocol while the other 2 received supportive management. Except for one $\mathrm{M}$-group case who received chemotherapy and AHCT, none of the other cases received AHCT because of the rapid clinical progression.

\section{Cyclin E2 expression is associated with high proliferation and poor outcome in TNKLPD}

Of the 4 cases that were profiled, 2 cases belonged to Pgroup (polymorphic infiltrate) and the other 2 were in $\mathrm{M}$ group (monomorphic infiltrate). Since the 2 groups showed significant difference in survival, we compared the genes differentially expressed between the two groups in order to identify targets that are associated with poor outcome. A total of 74 genes showed a 4-fold difference in expression between the 2 groups, of which 34 were upregulated (including CCNE2/cyclin E2) and 40 were downregulated in the group with shorter survival (Figure 3).

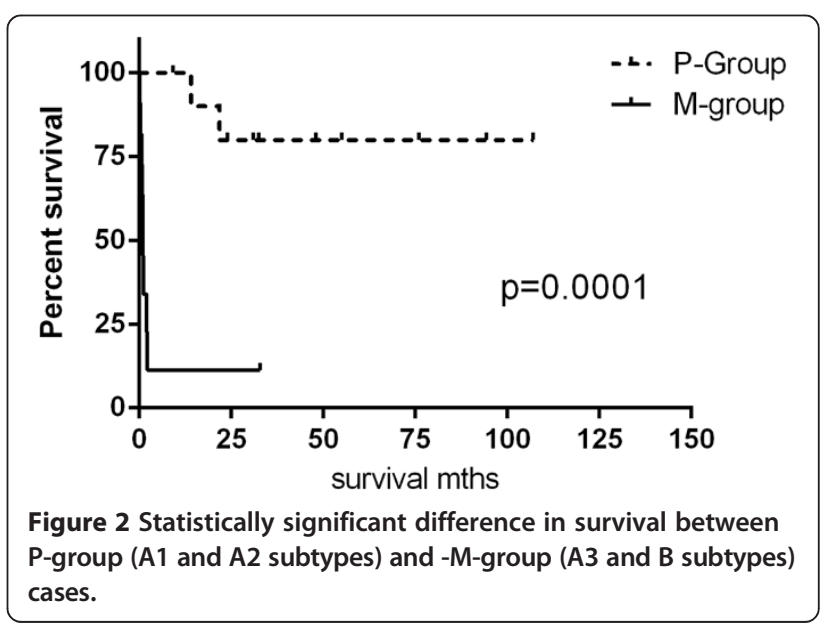


In order to validate our GEP findings, we performed immunohistochemistry on a few selected genes of interest and we found that there is a significant overexpression of cyclin E2 protein in M-group patients compared to P-group (median expression of $42 \%$ compared with $8 \%, \mathrm{p}=0.0005$ ) (Figure 4A, Table 3). There is also a statistically significant difference in survival between patients with low vs high cyclin E2 expression (median survival not yet reached vs 1.16 months, $\mathrm{p}=0.0002$ )
(Figure 4B) suggesting a potential role of cyclin E2 as an adverse prognostic marker in TNKLPD. Interestingly, only one case in P-group (case 11) had a high cyclin E2 expression and this patient also died of disease less than 2 years after diagnosis. Since cyclin E2 has an important role in cell cycle progression, we also performed Ki-67/ CD3 double stain to determine if the upregulation of cyclin E2 in M-group of TNKLPD correlated with a higher proliferation rate in this group. Indeed, we found

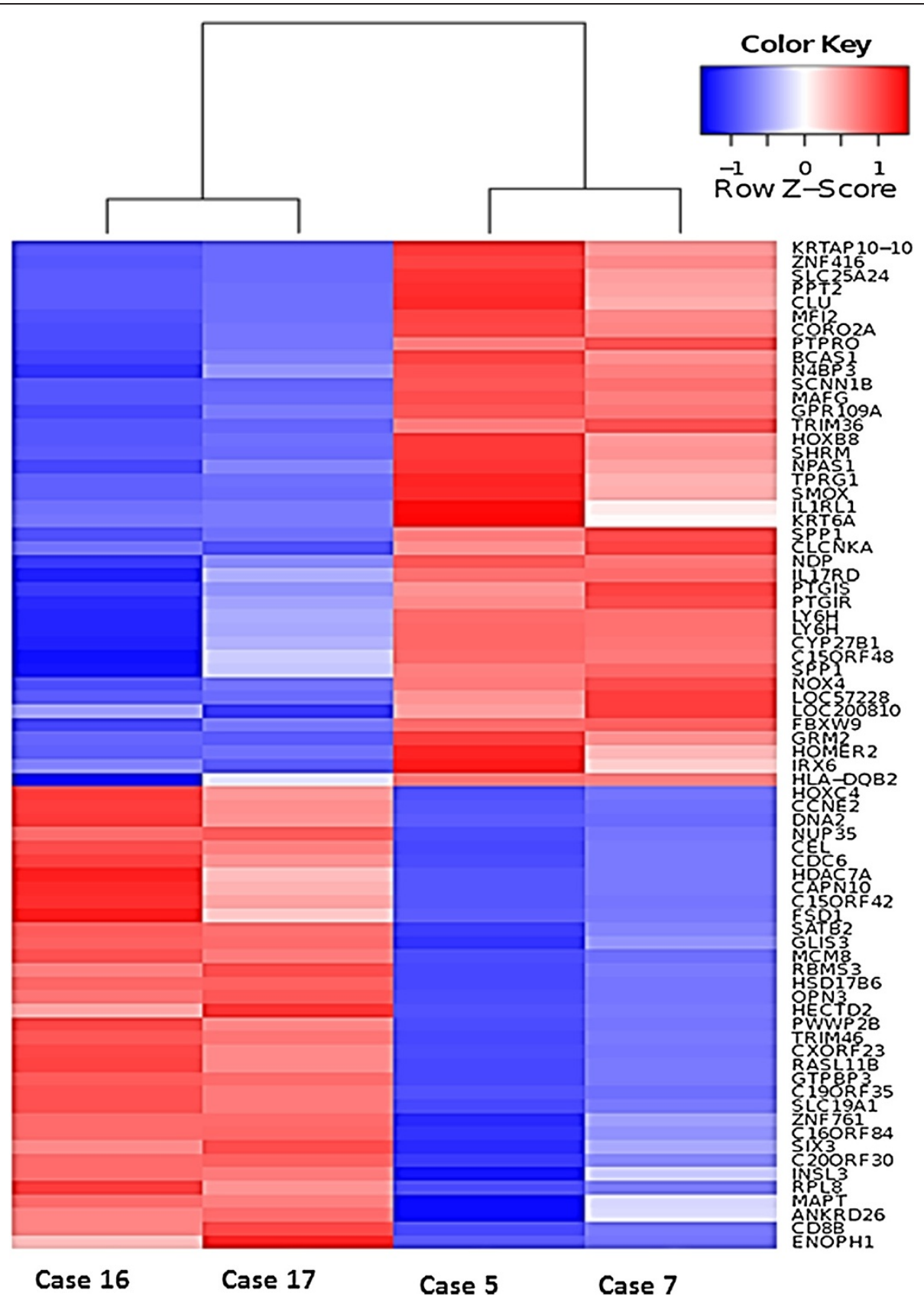

Figure 3 Differentially expressed genes between P-group and M-group patients with TNKLPD. 

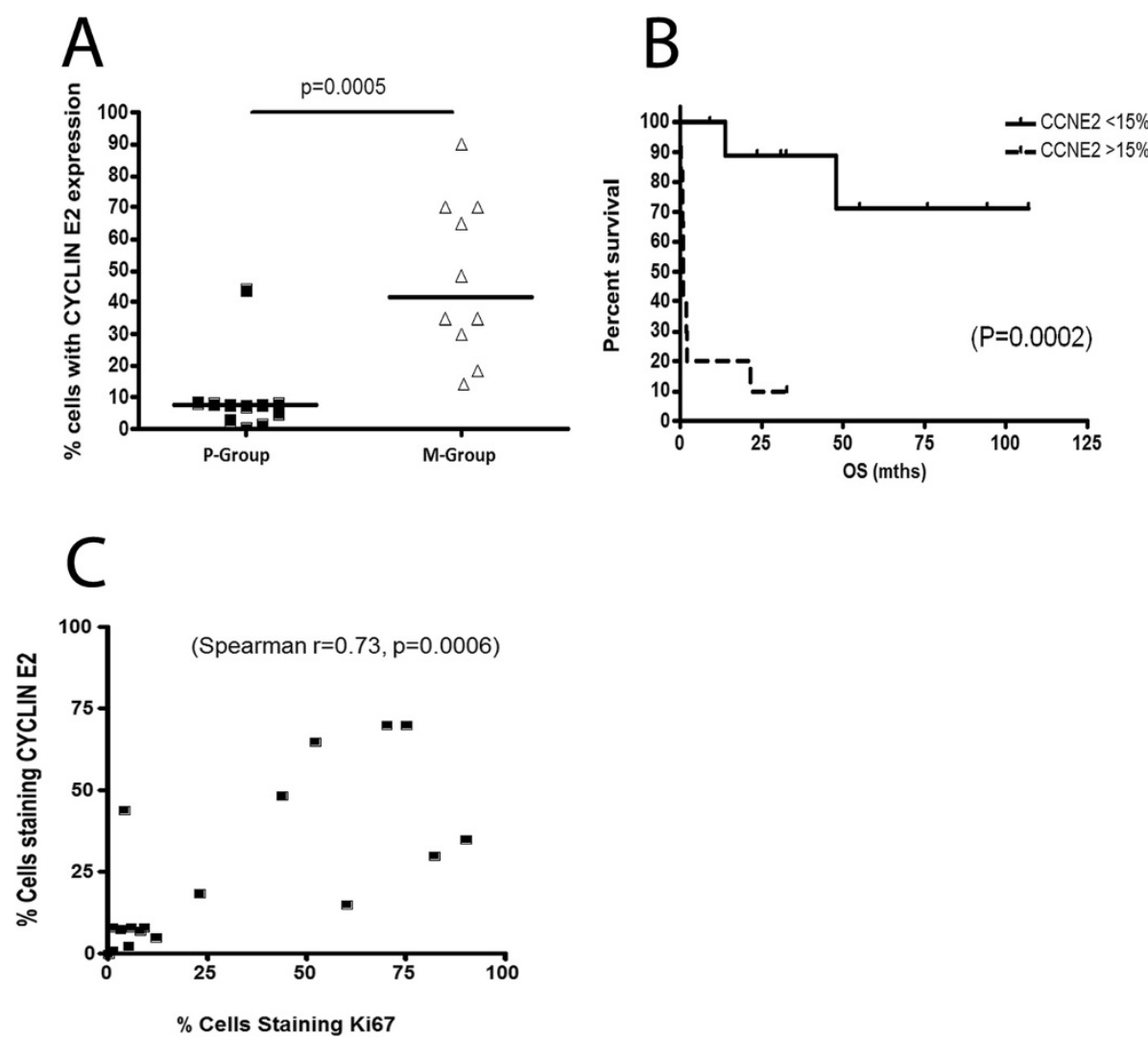

Figure 4 Cyclin E2 expression was associated with high proliferation rate and poor outcome in TNKLPD. Significantly higher cyclin E2 expression was present in M-group compared to P-group patients ( $\mathbf{A}$, median expression of $42 \%$ compared with $8 \%, p=0.0005)$. Patients with high tumor cyclin E2 expression of 15\% or greater had statistically significant shorter survival compared with those with low cyclin E2 expression less than $15 \%(\mathbf{B}, p=0.0002)$. High cyclin E2 expression is highly correlated with high Ki-67 proliferation rate $(\mathbf{C}$, spearman $r=0.73, p=0.0006)$.

a significant correlation between cyclin E2 and Ki-67 with spearman correlation $\mathrm{r}=0.73, \mathrm{p}=0.0006$ (Figure 4C, Table 3, Figure 5). The IHC of other selected genes were not included because of technical difficulty in performing double stains in TNKLPD tissue samples which included mostly bone marrow biopsy sections.

\section{Discussion}

Chronic active EBV infection of T/NK type (CAEBV-T/ $\mathrm{NK}$ ), aggressive NK cell leukemia (ANKL) in children and systemic EBV + T-cell lymphoproliferative disorders (LPD) of childhood are a group of systemic EBV-associated T/ NK cell LPD that is prevalent in children and young adults in Asia, Native Americans in Mexico and South America $[12,20]$. Although rare, this is an important group of disease to study because very little is known about the disease pathogenesis, and they are often aggressive in behavior with no effective treatment. Furthermore, biologic markers useful in predicting patient outcome have also not been identified. This is attributed to the rarity of the disease and limited availability of tissue because most of the tissue samples from such patients are bone marrow biopsies containing scanty amount of tumor of suboptimal quality secondary to decalcification that is required for the processing of bone marrow tissue samples.

In this study, we describe 22 cases of TNKLPD in Singapore and Japan which includes CAEBV-T/NK and systemic EBV + T-cell LPD in childhood. Although TNKLPD affects mainly children and young adults, two of our cases occurred in patients older than 30 years, similar to observations from previous studies that this disease can occasionally occur in older adults $[4,13,14]$. There were no aggressive NK cell leukemia (ANKL) and all our TNKLPD with fulminant clinical course (Ohshima B subtype) were of $\mathrm{T}$ cell origin and classified as systemic EBV-positive $\mathrm{T}$ cell LPD of childhood. The lack of systemic fulminant TNKLPD disease of NK origin may be due to more comprehensive testing for TCR clonality in combination with immunohistochemistry for the expression of $T C R \alpha \beta$ and $\mathrm{TCR} \gamma \delta$. Since antibodies for TCR $\alpha \beta$ and TCR $\gamma \delta$ for formalin fixed paraffin sections are only available in the recent years, it is possible that some of the ANKL diagnosed in the past may actually be of $\mathrm{T}$ cell origin due to limited T cell assessment. 
Table 3 Immunohistochemical expression of cyclin E2 and Ki67 in CD3-positive tumor population of TNKLPD

\begin{tabular}{|c|c|c|c|c|c|c|c|}
\hline Case No. & Diagnosis & Ohshima category & Histology & Cyclin E2 (\%) & Ki67 (\%) & Follow up & OS (mth) \\
\hline 1 & CAEBV & A1 & $P$ & 3 & 5 & Alive & 55 \\
\hline 2 & CAEBV & A1 & $P$ & 8 & NA & Dead (AHCT complications) & 48 \\
\hline 3 & CAEBV & A1 & $P$ & 0 & 0 & Alive & 6 \\
\hline 4 & STLPDC & A2 & $P$ & 8 & 6 & Alive & 64 \\
\hline 5 & STLPDC & A2 & $P$ & 1 & 1 & Alive cgraft rejection) & 95 \\
\hline 6 & STLPDC & A2 & $P$ & 5 & 12 & Alive & 31 \\
\hline 7 & STLPDC & $\mathrm{A} 2$ & $P$ & 8 & 3 & Dead & 14 \\
\hline 8 & CAEBV vs STLPDC & $\mathrm{A} 1$ or $\mathrm{A} 2$ & $P$ & 8 & 1 & Alive & 94.4 \\
\hline 9 & CAEBV vs STLPDC & $\mathrm{A} 1$ or $\mathrm{A} 2$ & $P$ & 7 & 8 & Alive & 9.2 \\
\hline 10 & CAEBV vs STLPDC & $\mathrm{A} 1$ or $\mathrm{A} 2$ & $P$ & 8 & 9 & Alive & 32.5 \\
\hline 11 & CAEBV vs STLPDC & $\mathrm{A} 1$ or $\mathrm{A} 2$ & $P$ & 44 & 4 & Dead & 21.8 \\
\hline 12 & STLPDC & A3 & M & 90 & NA & Dead & 0.4 \\
\hline 13 & STLPDC & A3 & M & 70 & 75 & Alive & 21 \\
\hline 14 & STLPDC & B & M & 49 & 44 & Dead & 0.83 \\
\hline 15 & STLPDC & B & M & 35 & NA & Dead & 2 \\
\hline 16 & STLPDC & B & M & 70 & 70 & Dead & 1 \\
\hline 17 & STLPDC & B & M & 15 & 60 & Dead & 1.16 \\
\hline 18 & STLPDC & B & M & 30 & 82 & NA & NA \\
\hline 19 & STLPDC & B & M & 35 & 90 & Dead & 1 \\
\hline 20 & STLPDC & B & M & 18 & 23 & Dead & 0.1 \\
\hline 21 & STLPDC & B & M & 65 & 52 & Dead & 2.2 \\
\hline 22 & favour STLPDC & $A 3$ or $B$ & M & NA & NA & Dead & 0.7 \\
\hline
\end{tabular}

Abbreviations: NA not available, $m$ th months, $P$ polymorphic, $M$ monomorphic.

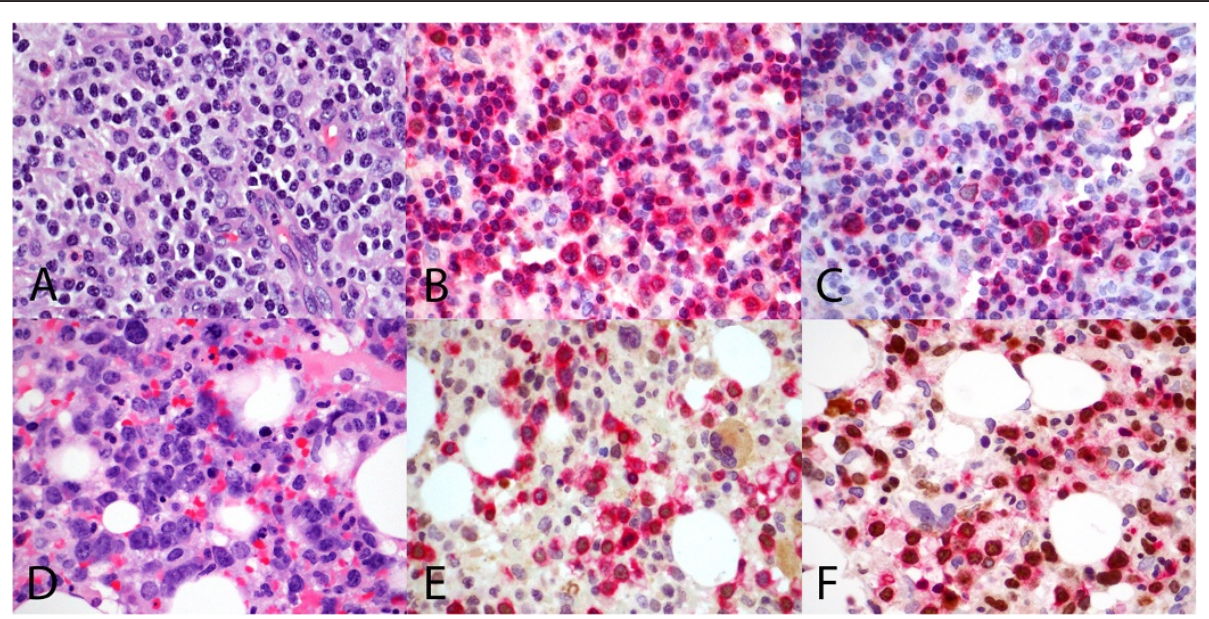

Figure 5 Immunohistochemical expression of cyclin E2 protein and Ki67 proliferation rate in M-group compared to P-group cases. Case 7 with type A2 disease and polymorphic morphology (P-group) (A, H\&E original magnification 600X) showing low cyclin E2 expression (B, CD3/cyclin E2 double stain, original magnification 600x) and low Ki67 proliferation (C, CD3/Ki67 double stain, original magnification 600x). Case 19 with type B disease and monomorphic morphology (M-group) (D, H\&E original magnification 600x) with moderately high cyclin E2 expression (E, CD3/cyclin E2 double stain, original magnification 600x) and high Ki67 proliferation (F, CD3/Ki67 double stain). CD3 stains cell membrane/ cytoplasm red, and cyclin E2 and Ki67 stain nucleus brown. All photographs were taken with a DP20 Olympus camera (Olympus, Tokyo, Japan) using an Olympus BX41 microscope (Olympus). Images were acquired using DP Controller 2002 (Olympus) and processed using Adobe Photoshop version 5.5 (Adobe Systems, San Jose, CA, USA). 
We found that cases with monomorphic infiltrate (Mgroup) had significantly shorter survival compared to Pgroup cases with polymorphic infiltrate. In our study, all but one case, with a monomorphic infiltrate had an overall survival of less than 3 months. This highlights the importance of morphology in predicting outcome and that cases with frank malignant proliferation should be regarded as an aggressive lymphoma and treated as such. This is in line with the recommendation from the consensus meeting in NIH 2008 that the term "CAEBV" should not be applied to systemic LPDs that contain a frankly malignant infiltrate, ie. Ohshima A3 and B subtypes [12].

Recently, Quintanilla-Martinez et al. described 20 cases of Hydroa vacciniforme-like lymphoma (HVLL), which is also an EBV-positive T-cell lymphoproliferative disorder of childhood but with predominant cutaneous manifestations [21]. The authors found that although HVLL in general showed a favorable response to conservative therapy, there is a risk to develop systemic lymphoma and criteria such as presence of systemic symptoms, T-cell clonality, amount of EBV-positive cells, and/or density of the infiltrate do not help in predicting which patients will eventually progress to systemic disease [21]. Hence, the challenge remains to identify morphological or clinical markers to predict outcome or clinical progression. Importantly, in this study of EBV-associated T/NK LPD with primarily systemic presentation, our GEP data revealed cyclinE2 gene to be overexpressed in M-group compared to P-group patients. Using immunohistochemistry double stains, we found a corresponding higher protein expression of cyclin E2 in M-group patients and this is associated with poor outcome, thus supporting the validity of our GEP data. We further showed that the upregulation of cyclinE2 in patients with poor outcome is significantly correlated with a higher Ki67 proliferation rate.

The question of whether cyclin E2 is merely a link in the chain of events that lead to cell proliferation or whether it is a driving force in cell replication in TNKLPD is difficult to ascertain at present. In some neoplasms, cyclin E gene amplification and protein accumulation are late events $[22,23]$ whereas in other tumors an increase in cyclin $\mathrm{E}$ is observed early in the progression to malignancy $[24,25]$. Since cyclinE2 overexpression is associated with high Ki67 proliferation, monomorphic large cell morphology and poor outcome, it is tempting to speculate that the upregulation of cyclinE2 is merely a reflection of tumor progression in TNKLPD. However, one patient (case 11) in this study showed polymorphic morphology and low Ki67 proliferation, but high cyclinE2 expression. This patient had a fairly aggressive outcome and died of disease within 2 years of diagnosis, suggesting that cyclinE2 upregulation in some TNKLPD may not simply be due to proliferation and disease progression. It is also interesting to note that, unlike cyclin E1 and Ki67 which are expressed in most proliferating normal and tumor cells, cyclin E2 levels are often low to undetectable in non-transformed cells and increased significantly in tumor-derived cells [26]. Hence, it is possible that cycinE2 may be a more specific marker of tumor proliferation than Ki67.

\section{Conclusion}

We described 22 cases of TNKLPD with systemic presentation, including CAEBV of T/NK type and systemic $\mathrm{EBV}+\mathrm{T}$ cell LPD of childhood and found the presence of a monomorphic infiltrate and high cyclin E2 and Ki-67 expression to be associated with poor outcome in TNKLPD, which is a rare disease with no known markers of prognostic significance to date. Whether cyclin E2 alone or in combination with Ki67 and morphology allows better prognostic stratification than standard morphology requires further study with larger sample size.

\section{Additional files}

Additional file 1: Comparison of the proposed nomenclature of EBV-positive T/NK lymphoproliferative disorder (LPD) with systemic presentation.

Additional file 2: Summary of immunohistochemical double stain conditions.

\section{Competing interests}

The authors declare that they have no competing interests.

\section{Authors' contributions}

S-BN and W-JC conceived and designed the study, analyzed/interpreted data, and wrote the paper. S-BN performed $\mathrm{IHC}$ scoring and reviewed cases. $\mathrm{KO}$ and HM reviewed cases. VS and S-NC performed experiments. GH performed bioinformatics analysis. SW, H-CC, AE-JY, T-CQ, L-PK, P-LT collected clinical and pathological data. All authors read and approved the final manuscript.

\section{Acknowledgments}

S.-B.N. is supported by National Medical Research Council Transition Award (grant number R-179-000-055-511). This work is partly supported by grant from the Ministry of Education (MOE) Academic Research Fund (AcRF) Tier 1 (grant number R-179-000-046-112).

W.-J.C. is supported by NMRC Clinician Scientist Investigator award. This work is partly supported by Singapore Cancer Syndicate Grant, and the National Research Foundation Singapore and the Singapore Ministry of Education under the Research Centers of Excellence initiative.

\section{Author details}

${ }^{1}$ Department of Pathology, National University Cancer Institute of Singapore, National University Health System, Yong Loo Lin School of Medicine, Cancer Science Institute of Singapore, National University of Singapore, Singapore, Singapore. ${ }^{2}$ Department of Pathology, Kurume University, Asahimati 67, Kurume 830-0011, Japan. ${ }^{3}$ Department of Pathology, National University Health System, Singapore, Singapore. ${ }^{4}$ Cancer Science Institute of Singapore, National University of Singapore, Singapore, Singapore. ${ }^{5}$ Department of Paediatrics, National University Health System, Singapore, Singapore.

${ }^{6}$ Department of Haematology-Oncology, National University Health System, Singapore, Singapore. ${ }^{7}$ Department of Haematology-Oncology, National University Cancer Institute of Singapore, National University Health System, Yong Loo Lin School of Medicine, Cancer Science Institute of Singapore, 1E, Kent Ridge Rd, Singapore 119228, Singapore. ${ }^{8}$ Department of Pathology, 
National University Hospital, 5 Lower Kent Ridge Road, Main Building, Level 3, Singapore 119074, Singapore.

Received: 27 August 2014 Accepted: 14 October 2014

Published online: 05 December 2014

\section{References}

1. Quintanilla-Martinez L, Kimura H, Jaffe ES: EBV-positive T-cell lymphoproliferative disorders of childhood. In WHO Classification of Tumours of Haematopoietic and Lymphoid Tissues. 4th edition. Edited by Swerdlow SH, Campo E, Harris NL, Jaffe ES, Pileri SA, Stein H, Thiele J, Vardiman JW. Lyon: IRAC Press; 2008:278-280.

2. Ko YH, Jaffe ES: Epstein-Barr Virus-Positive Systemic T-Lymphoproliferative Disorders and Related Lymphoproliferations of Childhood. In Hematopathology. Edited by Jaffe ES, Harris NL, Vardiman JW, Campo E, Arber DA. Philadelphia, PA: Elsevier Saunders; 2011:492-505.

3. Fujiwara S, Kimura H, Imadome K, Arai A, Kodama E, Morio T, Shimizu N, Wakiguchi $\mathrm{H}$ : Current research on chronic active Epstein-Barr virus infection in Japan. Pediatr Int 2014, 56:159-166.

4. Kimura H, Ito Y, Kawabe S, Gotoh K, Takahashi Y, Kojima S, Naoe T, Esaki S, Kikuta A, Sawada A, Kawa K, Ohshima K, Nakamura S: EBV-associated T/NK-cell lymphoproliferative diseases in nonimmunocompromised hosts: prospective analysis of 108 cases. Blood 2012, 119:673-686.

5. Suzuki K, Ohshima K, Karube K, Suzumiya J, Ohga S, Ishihara S, Tamura K, Kikuchi M: Clinicopathological states of Epstein-Barr virus-associated T/NK-cell lymphoproliferative disorders (severe chronic active EBV infection) of children and young adults. Int I Oncol 2004, 24:1165-1174.

6. Kimura H, Morishima T, Kanegane H, Ohga S, Hoshino Y, Maeda A, Imai S, Okano M, Morio T, Yokota S, Tsuchiya S, Yachie A, Imashuku S, Kawa K, Wakiguchi H, Japanese Association for Research on Epstein-Barr V, Related D: Prognostic factors for chronic active Epstein-Barr virus infection. J Infect Dis 2003, 187:527-533.

7. Ohshima K, Suzumiya J, Sugihara M, Nagafuchi S, Ohga S, Kikuchi M: Clinicopathological study of severe chronic active Epstein-Barr virus infection that developed in association with lymphoproliferative disorder and/or hemophagocytic syndrome. Pathol Int 1998, 48:934-943.

8. Quintanilla-Martinez L, Kumar S, Fend F, Reyes E, Teruya-Feldstein J, Kingma DW, Sorbara L, Raffeld M, Straus SE, Jaffe ES: Fulminant EBV(+) T-cell lymphoproliferative disorder following acute/chronic EBV infection: a distinct clinicopathologic syndrome. Blood 2000, 96:443-451.

9. Su IJ, Chen RL, Lin DT, Lin KS, Chen CC: Epstein-Barr virus (EBV) infects T lymphocytes in childhood EBV-associated hemophagocytic syndrome in Taiwan. Am J Pathol 1994, 144:1219-1225.

10. Kikuta H, Sakiyama Y, Matsumoto S, Oh-Ishi T, Nakano T, Nagashima T, Oka T, Hironaka T, Hirai K: Fatal Epstein-Barr virus-associated hemophagocytic syndrome. Blood 1993, 82:3259-3264.

11. Kimura H, Hoshino Y, Kanegane H, Tsuge I, Okamura T, Kawa K, Morishima T: Clinical and virologic characteristics of chronic active Epstein-Barr virus infection. Blood 2001, 98:280-286.

12. Cohen JI, Kimura H, Nakamura S, Ko YH, Jaffe ES: Epstein-Barr virus-associated lymphoproliferative disease in non-immunocompromised hosts: a status report and summary of an international meeting, 8-9 September 2008. Ann Oncol 2009, 20:1472-1482.

13. Ohshima K, Kimura H, Yoshino T, Kim CW, Ko YH, Lee SS, Peh SC, Chan JK: Proposed categorization of pathological states of EBV-associated T/natural killer-cell lymphoproliferative disorder (LPD) in children and young adults: overlap with chronic active EBV infection and infantile fulminant EBV T-LPD. Pathol Int 2008, 58:209-217.

14. Ko Y-H, Kim H-Y, Oh Y-H, Park G, Lee S-S, Huh J, Kim C-W, Kim I, Ng S-B, Tan S-Y, Chuang S-S, Nakamura N, Yoshino T, Nagamura S, Kimura H, Ohshima K: EBV-associated T and NK cell lymphoproliferative disorders: consensus report of the 4th Asian hematopathology workshop. J Hematopathol 2012, 5:319-324.

15. Henter J, Horne A, Arico M, Egeler RM, Filipovich AH, Imashuku S, Ladisch S, McClain K, Webb D, Winiarski J, Janka G: HLH-2004: diagnostic and therapeutic guidelines for hemophagocytic lymphohistiocytosis. Pediatr Blood Cancer 2007, 48:124-131.

16. Fan JB, Yeakley JM, Bibikova M, Chudin E, Wickham E, Chen J, Doucet D, Rigault P, Zhang B, Shen R, McBride C, Li HR, Fu XD, Oliphant A, Barker DL, Chee MS: A versatile assay for high-throughput gene expression profiling on universal array matrices. Genome Res 2004, 14:878-885.
17. Bibikova M, Talantov D, Chudin E, Yeakley JM, Chen J, Doucet D, Wickham E, Atkins D, Barker D, Chee M, Wang Y, Fan JB: Quantitative gene expression profiling in formalin-fixed, paraffin-embedded tissues using universal bead arrays. Am J Pathol 2004, 165:1799-1807.

18. Ng SB, Selvarajan V, Huang G, Zhou J, Feldman AL, Law M, Kwong YL, Shimizu N, Kagami Y, Aozasa K, Salto-Tellez M, Chng WJ: Activated oncogenic pathways and therapeutic targets in extranodal nasal-type NK/T cell lymphoma revealed by gene expression profiling. J Pathol 2010, 223:496-510.

19. Tusher VG, Tibshirani R, Chu G: Significance analysis of microarrays applied to the ionizing radiation response. Proc Natl Acad Sci U S A 2001, 98:5116-5121.

20. Ng SB, Khoury JD: Epstein-Barr virus in lymphoproliferative processes: an update for the diagnostic pathologist. Adv Anat Pathol 2009, 16:40-55.

21. Quintanilla-Martinez L, Ridaura C, Nagl F, Saez-de-Ocariz M, Duran-McKinster C, Ruiz-Maldonado R, Alderete G, Grube P, Lome-Maldonado C, Bonzheim I, Fend F: Hydroa vacciniforme-like lymphoma: a chronic EBV + lymphoproliferative disorder with risk to develop a systemic lymphoma. Blood 2013, 122:3101-3110.

22. Akama Y, Yasui W, Yokozaki H, Kuniyasu H, Kitahara K, Ishikawa T, Tahara E: Frequent amplification of the cyclin $\mathrm{E}$ gene in human gastric carcinomas. Jpn J Cancer Res 1995, 86:617-621.

23. Yasui W, Kudo Y, Semba S, Yokozaki H, Tahara E: Reduced expression of cyclin-dependent kinase inhibitor p27Kip1 is associated with advanced stage and invasiveness of gastric carcinomas. Jpn J Cancer Res 1997, 88:625-629.

24. Bito T, Ueda M, Ito A, Ichihashi M: Less expression of cyclin $\mathrm{E}$ in cutaneous squamous cell carcinomas than in benign and premalignant keratinocytic lesions. J Cutan Pathol 1997, 24:305-308.

25. Donnellan R, Chetty R: Cyclin E in human cancers. FASEB J 1999, 13:773-780.

26. Gudas JM, Payton M, Thukral S, Chen E, Bass M, Robinson MO, Coats S: Cyclin E2, a novel G1 cyclin that binds Cdk2 and is aberrantly expressed in human cancers. Mol Cell Biol 1999, 19:612-622.

\section{doi:10.1186/s13023-014-0165-x}

Cite this article as: $\mathrm{Ng}$ et al:: Prognostic implication of morphology, cyclinE2 and proliferation in EBV-associated T/NK lymphoproliferative disease in non-immunocompromised hosts. Orphanet Journal of Rare Diseases 2014 9:165.

\section{Submit your next manuscript to BioMed Central and take full advantage of:}

- Convenient online submission

- Thorough peer review

- No space constraints or color figure charges

- Immediate publication on acceptance

- Inclusion in PubMed, CAS, Scopus and Google Scholar

- Research which is freely available for redistribution

Submit your manuscript at www.biomedcentral.com/submit
C Biomed Central 